Research Journal of Applied Sciences, Engineering and Technology 5(9): 2754-2759, 2013

DOI:10.19026/rjaset.5.4802

ISSN: 2040-7459; e-ISSN: 2040-7467

(C) 2013 Maxwell Scientific Publication Corp.

Submitted: September 25, $2012 \quad$ Accepted: November 08, 2012

Published: March 20, 2013

\title{
Research Article \\ Diversity Gain and Coding Performance of OST-CPM System in Spatially Correlated Channels
}

\author{
Wen-Li Shen and An-Ding Wang \\ Colleges of Information and Electronic Engineering, Zhejiang Gongshang University, Hangzhou, China
}

\begin{abstract}
The Chernoff bound on Pair-Wise Error Probability (PWEP) performance of orthogonal space-time coded CPM (OST-CPM) systems is investigated, for two transmit antennas, over spatially correlated channels. Approximate Chernoff bound for high Signal-to-Noise Ratio (SNR) is derived to evaluate the diversity gain and coding performance in correlated channels. The achievable diversity gain of this system decreases due to the signal correlation between the antennas. Simulation results show that the error performance over a correlated channel is degraded when the correlation coefficient increases. And the penalty on the code performance increases a lot in fully correlated channels. It can also be seen that the diversity gain decreases when the channel is fully correlated, which matches well with the theoretical analysis. The upper bounds can be looser when diversity order decreases.
\end{abstract}

Keywords: Correlated channel, OST-CPM, pair-wise error probability

\section{INTRODUCTION}

Space-time coding has shown considerable promise for reliable transmission over wireless fading channels by efficiently employing diversity. It is an efficient scheme to overcome the effects of multiple-path fading and increase the data rate for wireless communication systems (Foschini, 1996; Altamonte, 1998; Tarokh et al., 1998, 1999a; Naguib et al., 1998; Baro et al., 2000). An Orthogonal Space-Time coding (OST) design was proposed by Altamonte (1998), which improves the capacity of wireless communication systems. This scheme achieves a full diversity gain with a simple maximum-likelihood (ML) decoding of multiple symbols to be reduced into ML decoding of individual symbols, due to the orthogonality. This idea has been extended to a general number of transmit antennas (Tarokh et al., 1999b). The Continuous Phase Modulation (CPM) has the characteristics of constant envelope and phase continuity. Therefore CPM has become an attractive scheme for the data transmission over both bandwidth and power limited links such as mobile satellite communications (Aulin et al., 1981; Proakis, 1983; Anderson et al., 1986).

Combining CPM with space-time coding (STCCPM) can provide better performance in wireless communications. It can not only provide power and bandwidth efficiency but also achieve diversity and coding gains without sacrificing data rate or bandwidth (Zhang and Fitz, 2003; Wang and Xia, 2004; Wang et al., 2005; Zajic and Stuber, 2009; Wang and
Abeysekera, 2011; Hesse et al., 2011). Zhang and Fitz (2003) recently proposed a trellis-coded space-time CPM (TC-ST-CPM) system, where design rules and code constructions were derived to achieve full diversity. Wang and Xia (2004) and Wang et al. (2005) extended Alamouti's orthogonal encoding criterion to CPM signals and designed orthogonal space-time coded CPM (OST-CPM) systems. Modifications during every symbol period were made to ensure the phase continuity and orthogonality of the CPM waveforms transmitted over different antennas.

Chernoff bounds on Pair-Wise Error Probabilities (PWEP) have been used to analyze the performance of Multiple Input Multiple Output (MIMO) wireless communication systems (Hochwald and Marzetta, 2000; Hughes, 2000). In multiple antenna systems, insufficient antenna spacing, angle spread and the lack of rich scattering may cause spatial correlation between antennas. Bolcskei and Raj (2000) and Uysal and Georghiades (2001) studied the effect of spatial correlation on the PWEP of the space-time code in slow Rayleigh fading channels. In this study, we derive Chernoff bound for Maximum Likelihood Sequence Detection (MLSD) algorithm of the OST-CPM system in a correlated quasi-static Rayleigh-fading channel. Approximate Chernoff bound and achievable diversity gain is derived for high scattering Signal-to-Noise Ratio (SNR). The Frame Error Rate (FER) performances of OST-CPM systems in correlated and uncorrelated channels are simulated and compared with each other.

\footnotetext{
Corresponding Author: Wen-Li Shen, College of Information and Electronic Engineering, Zhejiang Gongshang University, Hangzhou, China

This work is licensed under a Creative Commons Attribution 4.0 International License (URL: http://creativecommons.org/licenses/by/4.0/)
} 


\section{SYSTEM MODEL}

In this study, a wireless communication system with $n_{T}$ transmit and $n_{R}$ receive antennas is considered. For simplification we just focus on the baseband equivalent block diagram of the OST-CPM system which is shown in Fig. 1.

We define an $n_{T} \times L$ space-time codeword matrix $X$ as the input of space-time coded modulator, obtained by arranging the transmitted sequence in an array as:

$$
\boldsymbol{X}=\left[\boldsymbol{x}_{1}, \boldsymbol{x}_{2}, \cdots, \boldsymbol{x}_{n_{T}}\right]^{T}=\left[\begin{array}{cccc}
x_{1,1} & x_{1,2} & \cdots & x_{1, L} \\
x_{2,1} & x_{2,2} & \cdots & x_{2, L} \\
\vdots & \vdots & \ddots & \vdots \\
x_{n_{T}, 1} & x_{n_{T}, 2} & \cdots & x_{n_{T}, L}
\end{array}\right]
$$

where, the $i$-th row $\boldsymbol{x}_{i}=\left[x_{i, 1}, x_{i, 2}, \ldots, x_{i, L}\right]$ is the data sequence transmitted from the $i$-th transmit antenna. Then the OST-CPM signals are simultaneously transmitted from the $n_{T}$ transmit antennas.

The transmitted signal at the $i$-th antenna can be represented as:

$$
\begin{aligned}
& s_{i}\left(t, \boldsymbol{x}_{i}\right)=\sqrt{\frac{E_{s}}{T}} \exp \left\{j \phi\left(t, \boldsymbol{x}_{i}\right)\right\}, i=1,2, \cdots, n_{T} \\
& \phi\left(t, \boldsymbol{x}_{i}\right)=2 \pi h \sum_{n=0}^{k} x_{i, n} q(t-n T), k T \leq t<(k+1) T
\end{aligned}
$$

where,

$E_{\mathrm{s}} \quad=$ The symbol energy

$T \quad=$ The symbol period

$\phi\left(t, x_{i}\right) \quad=$ The carried phase information

$h \quad=$ The modulation index

$q(t)=\int_{-\infty}^{t} g(\tau) d \tau=$ The phase smoothing response function

$g(t) \quad=$ The pulse shaping function, which is nonzero only at the limited time period $0 \leq t \leq L T$ ( $L$ is the modulation memory) and it decides the smoothness of the transmitted phase. Then the transmitted signals from the $n_{T}$ transmit antennas at time $t$ can be represented by a $n_{T} \times 1$ column vector $\mathrm{S}_{t}$;

$$
\boldsymbol{S}_{t}=\left[s_{1}\left(t, \boldsymbol{x}_{1}\right), s_{2}\left(t, \boldsymbol{x}_{2}\right), \cdots, s_{n_{T}}\left(t, \boldsymbol{x}_{n_{T}}\right)\right]^{T}
$$

Wang et al. (2005) extended Alamouti's encoding rule to CPM, where a design methodology was presented such that the signals $s_{1}\left(t, \boldsymbol{x}_{1}\right)$ and $s_{2}\left(t, \boldsymbol{x}_{2}\right)$ from the two transmit antennas over two symbol periods are orthogonal, i.e., the rows of the matrix:

$$
\left[\begin{array}{ll}
s_{1}\left(t, \boldsymbol{x}_{1}\right) & s_{1}\left(t+T, \boldsymbol{x}_{1}\right) \\
s_{2}\left(t, \boldsymbol{x}_{2}\right) & s_{2}\left(t+T, \boldsymbol{x}_{2}\right)
\end{array}\right]
$$

are orthogonal for each $t$. In this study, full response CPM signals $(L=1)$ are mainly considered. In order to ensure the orthogonality between two waveforms $s_{1}(t$, $\left.x_{1}\right)$ and $s_{2}\left(t, x_{2}\right)$, a second phase smoothing response function $q_{0}(t)$ is introduced with $q(0)=q_{0}(0)=0, q_{0}(T)$ $=q_{0}(T)=1 / 2$.

For wireless mobile communications, each link from a transmit antenna to a receive antenna can be modeled by flat fading, if we assume that the channel is memory less. The MIMO channel with $n_{T}$ transmit and $n_{R}$ receive antennas can be represented by an $n_{R} \times n_{T}$ channel matrix $H$. At time t, the channel matrix $\mathrm{H}$ is given by:

$$
\boldsymbol{H}_{t}=\left[\begin{array}{cccc}
h_{1,1}(t) & h_{1,2}(t) & \cdots & h_{1, n_{R}}(t) \\
h_{2,1}(t) & h_{2,2}(t) & \cdots & h_{2, n_{R}}(t) \\
\vdots & \vdots & \ddots & \vdots \\
h_{n_{T}, 1}(t) & h_{n_{T}, 2}(t) & \cdots & h_{n_{T}, n_{R}}(t)
\end{array}\right]
$$

where $h_{i, j}(t)$ is the correlated fading coefficient for the path from the $i$-th transmit antenna to the $j$-th receive antenna and is modeled as a complex Gaussian random variable with zero mean and variance $\sigma^{2}$, implying that the amplitude of the path coefficients are modeled as Rayleigh fading. In terms of the coefficient variation speed, we only consider quasi-static fading channels. It is assumed that the fading coefficients are constant during a frame and vary from one frame to another which means that the symbol period is small compared to the channel coherence time. So the fading coefficient $h_{i, j}(t)$ can be simplified as $h_{i, j}$.

A slightly less general but more useful model considers correlations on transmit and receive sides

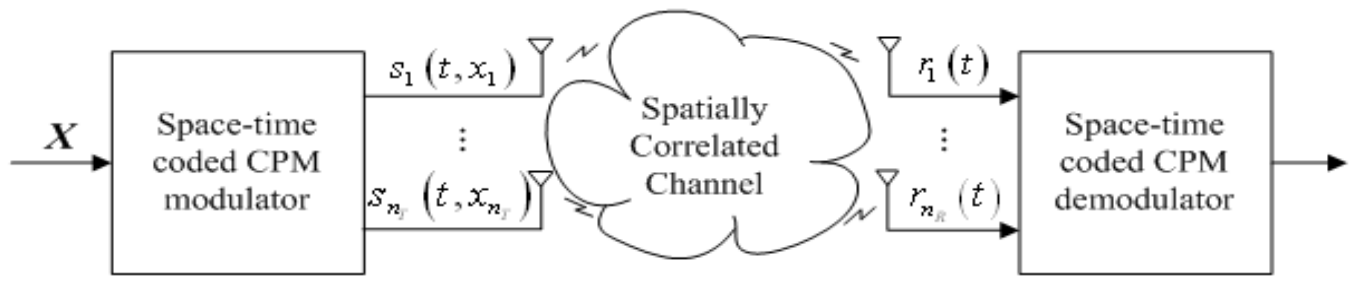

Fig. 1: Simplified base-band equivalent block diagram of ST-CPM system 
separately. The transmit correlation matrix is represented as $R_{T}$ while receive correlation matrix is as $R_{R}$. The coefficient matrix in correlated channels is represented as (Chuah et al., 2002):

$$
\boldsymbol{H}=\boldsymbol{R}_{\boldsymbol{R}}^{\frac{1}{2}} \boldsymbol{H}_{w} \boldsymbol{R}_{T}^{\frac{1}{2}}
$$

where, $H_{w}$ is a i.i.d. channel fading coefficients matrix.

At the receiver, the signal at each receive antenna is a noisy superposition of the $n_{T}$ transmitted signals degraded by channel fading. At time $t$, the received signals at the $j$-th antenna can be written as:

$$
r_{j}(t)=\sum_{i=1}^{n_{T}} h_{i, j} s_{i}\left(t, \boldsymbol{x}_{i}\right)+n_{j}(t), j=1,2, \cdots, n_{R}
$$

where, $n_{j}(t)$ is the noise component of the $j$-th receive antenna at time $t$, which is modeled as a zero-mean complex Gaussian random variable with power spectral density $N_{0}$ per dimension.

\section{PERFORMANCE ANALYSIS}

The optimum decoding and demodulation of OSTCPM system is Maximum Likelihood Sequence Detection (MLSD) algorithm, which could be expressed as:

$$
\hat{\boldsymbol{X}}=\underset{d}{\arg \min }\left\{\sum_{j=1}^{n_{R}} \int_{0}^{L T}\left|r_{j}(t)-\sum_{i=1}^{n_{T}} h_{i, j} s_{i}(t)\right|^{2} d t\right\}
$$

The Pair-Wise Error Probability (PWEP) conditioned on $\mathrm{H}$ can be approximated by Wang et al. (2005):

$$
P(\boldsymbol{X} \rightarrow \hat{\boldsymbol{X}} \mid \boldsymbol{H})=Q\left(\sqrt{\frac{E_{s}}{2 N_{0}} d^{2}[S(\boldsymbol{X}), S(\hat{\boldsymbol{X}})]}\right)
$$

where, $d^{2}[\boldsymbol{S}(\boldsymbol{X}), \boldsymbol{S}(\hat{\boldsymbol{X}})]$ is a modified Euclidean distance between the two space-time coded CPM signal matrices $S(X)$ and $\boldsymbol{S}(\hat{\boldsymbol{X}})$ :

$$
\text { Let } \Gamma=d^{2}[\boldsymbol{S}(\boldsymbol{X}), \boldsymbol{S}(\hat{\boldsymbol{X}})]
$$

$$
\begin{aligned}
& d^{2}[\boldsymbol{S}(\boldsymbol{X}), \boldsymbol{S}(\hat{\boldsymbol{X}})] \\
& =\sum_{j=1}^{n_{R}} \int_{0}^{L T}\left|\sum_{i=1}^{n_{T}} h_{i, j}\left[s_{i}\left(t, \boldsymbol{x}_{i}\right)-s_{i}\left(t, \hat{\boldsymbol{x}}_{i}\right)\right]\right|^{2} d t
\end{aligned}
$$

By using Graig's formula for the Gaussian Q function:

$$
Q(x)=\frac{1}{\pi} \int_{0}^{\frac{\pi}{2}} \exp \left\{-\frac{x^{2}}{2 \sin ^{2} \theta}\right\} d \theta
$$

we can rewrite the conditional PWEP:

$$
P(\boldsymbol{X} \rightarrow \hat{\boldsymbol{X}} \mid \boldsymbol{H})=\frac{1}{\pi} \int_{0}^{\frac{\pi}{2}} \exp \left\{-\frac{E_{s}}{4 N_{0} \sin ^{2} \theta} \Gamma\right\} d \theta
$$

In order to calculate the average PWEP, we average (13) with respect to the distribution of $\Gamma$. The average PWEP can be represented in terms of the moment generating function (MGF) of $\Gamma$, which is given by:

$$
M_{\Gamma}(s)=\int_{0}^{\infty} e^{s \Gamma} P_{\Gamma}(\Gamma) d \Gamma
$$

Thus the average PWEP can be represented as:

$$
\begin{aligned}
& P(\boldsymbol{X} \rightarrow \hat{\boldsymbol{X}}) \\
= & \frac{1}{\pi} \int_{0}^{\frac{\pi}{2}} E\left[\exp \left(-\frac{E_{s}}{4 N_{0} \sin ^{2} \theta} \Gamma\right)\right] d \theta \\
= & \frac{1}{\pi} \int_{0}^{\frac{\pi}{2}} \int_{0}^{\infty} \exp \left(-\frac{E_{s}}{4 N_{0} \sin ^{2} \theta} \Gamma\right) P_{\Gamma}(\Gamma) d \Gamma d \theta \\
= & \frac{1}{\pi} \int_{0}^{\frac{\pi}{2}} M_{\Gamma}\left(-\frac{E_{s}}{4 N_{0} \sin ^{2} \theta}\right) d \theta
\end{aligned}
$$

Equation (11) can be rewritten as:

$$
d^{2}[\boldsymbol{S}(\boldsymbol{X}), \boldsymbol{S}(\hat{\boldsymbol{X}})]=\operatorname{tr}\left(\boldsymbol{H B}(\boldsymbol{X}, \hat{\boldsymbol{X}}) \boldsymbol{H}^{H}\right)
$$

where $B\left(d_{1}, d_{2}\right)$ is a $n_{T} \times n_{T}$ signal difference matrix as shown in Eq. (19).

And:

$$
\Delta_{i}(t)=s_{i}\left(t, \boldsymbol{x}_{i}\right)-s_{i}\left(t, \hat{\boldsymbol{x}}_{i}\right)
$$

Therefore the PWEP can be represented as (Hedayat et al., 2005):

$$
\begin{gathered}
P(\boldsymbol{X} \rightarrow \hat{\boldsymbol{X}})=\frac{1}{\pi} \int_{0}^{\frac{\pi}{2}} \prod_{i=1}^{n_{T}} \prod_{j=1}^{n_{R}}\left(1+\frac{E_{s}}{4 N_{0} \sin ^{2} \theta} \mu_{i} \lambda_{j}\right)^{-1} d \theta \\
\leq \prod_{i=1}^{n_{T}} \prod_{j=1}^{n_{R}}\left(1+\frac{E_{s}}{4 N_{0}} \mu_{i} \lambda_{j}\right)^{-1} \\
\boldsymbol{B}(\boldsymbol{X}, \hat{\boldsymbol{X}})=\left[\begin{array}{cccc}
\int_{0}^{L T}\left|\Delta_{1}(t)\right|^{2} d t & \int_{0}^{L T} \Delta_{1}^{*}(t) \Delta_{2}(t) d t & \cdots & \int_{0}^{L T} \Delta_{l}^{*}(t) \Delta_{n_{T}}(t) d t \\
\int_{0}^{L T} \Delta_{1}(t) \Delta_{2}^{*}(t) d t & \int_{0}^{L T}\left|\Delta_{2}(t)\right|^{2} d t & \cdots & \int_{0}^{L T} \Delta_{2}^{*}(t) \Delta_{n_{T}}(t) d t \\
\vdots & \vdots & \ddots & \vdots \\
\int_{0}^{L T} \Delta_{1}(t) \Delta_{n_{T}}^{*}(t) d t & \int_{0}^{L T} \Delta_{2}(t) \Delta_{n_{T}}^{*}(t) d t & \cdots & \int_{0}^{L T}\left|\Delta_{n_{T}}(t)\right|^{2} d t
\end{array}\right]
\end{gathered}
$$


where $\mu_{i}$ and $\lambda_{j}$ are the Eigen values of matrices $\boldsymbol{B}(\boldsymbol{X}, \hat{\boldsymbol{X}}) R_{T}$ and $R_{R}$ respectively. Equation (18) is the Chernoff bound for the PWEP. When the SNR is high, the upper bound can be simplified as Tarokh et al. (1998):

$$
P(\boldsymbol{X} \rightarrow \hat{\boldsymbol{X}}) \leq\left(\frac{E_{s}}{4 N_{0}}\right)^{-r \hat{r}} \prod_{i=1}^{r} \prod_{j=1}^{\hat{r}}\left(\mu_{i} \lambda_{j}\right)^{-1}
$$

where, $r$ and $\hat{r}$ are the ranks of $\mathrm{B}(X, \hat{X}) \mathrm{R}_{\mathrm{T}}$ and $R_{R}$ respectively. $\mu_{\mathrm{i}}(\mathrm{i}=1,2, \ldots, \mathrm{r})$ and $\lambda_{\mathrm{ji}}(\mathrm{i}=1,2, \ldots, \mathrm{r})$ are the nonzero eigenvalues of $\mathrm{B}(X, \hat{X}) \mathrm{R}_{\mathrm{T}}$ and $R_{R}$. In the high-SNR regime, the diversity order of a pair of codewords of the OST-MCPM system is the exponent of SNR, i.e.:

$$
r \hat{r}=\operatorname{rank}\left(\boldsymbol{B}(\boldsymbol{X}, \hat{\boldsymbol{X}}) R_{T}\right) \cdot \operatorname{rank}\left(R_{R}\right)
$$

For the special case of fully correlated channel where $\left(R_{T}\right)_{i, j}=\left(R_{R}\right)_{i, j}=1$, the diversity order $r \hat{r}$ is 1 . For i.i.d. channels, the spatially correlated channels are $R_{T}=I_{n_{T}}, R_{R}=I_{n_{R}}$. Consequently, the achievable diversity gain could be represented as $r \hat{r}=\operatorname{rank}\left(\boldsymbol{B}(\boldsymbol{X}, \hat{\boldsymbol{X}}) R_{T}\right) \cdot n_{R}$

The typical scenario in the wireless channel environment is partial channel correlation. The channel correlation matrices $R_{T}$ and $R_{R}$ are nonnegative definite Hermitian matrices of a Toeplitz form. $R_{T}=R_{T}^{H}, \operatorname{det}\left(R_{T}\right)>0 \quad$ and $\quad R_{R}=R_{R}^{H}, \operatorname{det}\left(R_{R}\right)>0$. Obviously the correlation matrices $R_{T}$ and $R_{R}$ will always have full ranks. Thus the diversity order is the same as that in i.i.d. channels.

In order to achieve full diversity, $B(X, \hat{X})$ should be full rank. The waveform difference from the $n_{T}$ transmit antennas should be linearly independent over the complex field. That is to say, for any complex numbers $\boldsymbol{c}=\left[c_{1}, c_{2}, \ldots, c_{n_{T}}\right]$, there exists a $t$, such that (Wang et al., 2005):

$$
\sum_{i=1}^{n_{T}} c_{i} \Delta_{i}(t) \neq 0
$$

unless $c_{1}=c_{2}=\ldots=\mathrm{c}_{\mathrm{nT}}=0$.

Because the transmitted signals from the $n_{T}$ antennas are orthogonal, the transmitted signal matrix $\mathrm{S}$ $(t, X)$ is unitary. Thus it is easy to see that the OSTCPM satisfies the above condition of full rank. Therefore, in i.i.d. or partially correlated channels the OST-CPM system can achieve full diversity.

Finally we will analyze the encoding performance variation of the OST-CPM system when the MIMO channels are correlated. In independent MIMO fading channels, the correlation coefficient matrices $R_{T}$ and $R_{R}$ are unit with $n_{T}$ and $n_{R}$ nonzero eigenvalues respectively, which is:

$$
\mu_{i}^{\prime}\left(i=1,2, \cdots, n_{T}\right)=\lambda_{j}\left(j=1,2, \cdots, n_{R}\right)=1
$$

$B(X, \hat{X}) \mathrm{R}_{\mathrm{T}}$ and $R_{T}$ are independent and $R_{T}$ has full rank, thus the rank of $B(, X, \hat{X}) R_{T}$ is the same as the one of $\mathrm{B}(X, \hat{X}) \mathrm{R}_{\mathrm{T}}$. The product of $B(, X, \hat{X}) R_{T}$ all the nonzero eigenvalues $\mu_{i}(i=1,2, \ldots, r)$ of equals to the product of the nonzero eigenvalues $\mu_{i}^{\prime \prime}(i=1,2, \cdots, r) \quad$ of $\quad \boldsymbol{B}(\boldsymbol{X}, \hat{\boldsymbol{X}}) \quad$ and the ones $\mu_{i}^{\prime}\left(i=1,2, \cdots, n_{T}\right)$ of $R_{T}$ :

$$
\prod_{i=1}^{r} \mu_{i}=\prod_{i=1}^{r} \mu_{i}^{\prime \prime} \cdot \prod_{i=1}^{n_{T}} \mu_{i}^{\prime}=\prod_{i=1}^{r} \mu_{i}^{\prime \prime}
$$

and

$$
\prod_{i=1}^{n_{T}} \mu_{i}^{\prime}=1, \prod_{j=1}^{n_{R}} \lambda_{j}=1
$$

For independent MIMO channels, the PWEP upper bound of OST-CPM could be simplified to:

$$
P_{\text {full }}(\boldsymbol{X} \rightarrow \hat{\boldsymbol{X}}) \leq\left(\frac{E_{S}}{4 N_{0}}\right)^{-r n_{R}} \prod_{i=1}^{r}\left(\mu_{i}^{\prime \prime}\right)^{-1}
$$

When the MIMO channel is partial correlated, matrices $R_{T}$ and $R_{R}$ is full rank. The PWEP upper bound could be represented as:

$$
P_{\text {partial }}(\boldsymbol{X} \rightarrow \hat{\boldsymbol{X}}) \leq\left(\frac{E_{s}}{4 N_{0}}\right)^{-r n_{R}} \prod_{i=1}^{r} \prod_{i=1}^{n_{T}} \prod_{j=1}^{n_{R}}\left(\mu_{i}^{\prime} \mu_{i}^{\prime \prime} \lambda_{j}\right)^{-1}
$$

For full rank matrices $R_{T}$ and $R_{R}$ with Toeplitz form, all the eigenvalues $\mu_{i}^{\prime}\left(i=1,2, \cdots, n_{T}\right)$ and $\lambda_{\mathrm{j}}(\mathrm{i}=$ $\left.1,2, \ldots, \mathrm{n}_{\mathrm{R}}\right)$ are nonzero and the multiplication of all eigenvalues is smaller than 1 . Thus:

$$
\prod_{i=1}^{n_{T}}\left(\mu_{i}^{\prime \prime}\right)^{-1}>1, \prod_{j=1}^{n_{R}}\left(\lambda_{j}\right)^{-1}>1
$$

The PWEP upper bound is increased for Eq. (26) with independent MIMO channels. Finally we have deduced that the encoding performance of the OSTCPM system in partial correlated channels is degraded than in independent channels.

\section{SIMULATION RESULTS}

In this section, some simulation results are presented to evaluate the FER performances and the approximate Chernoff bounds of the MLSD over quasi-static Rayleigh fading channels. The FER 


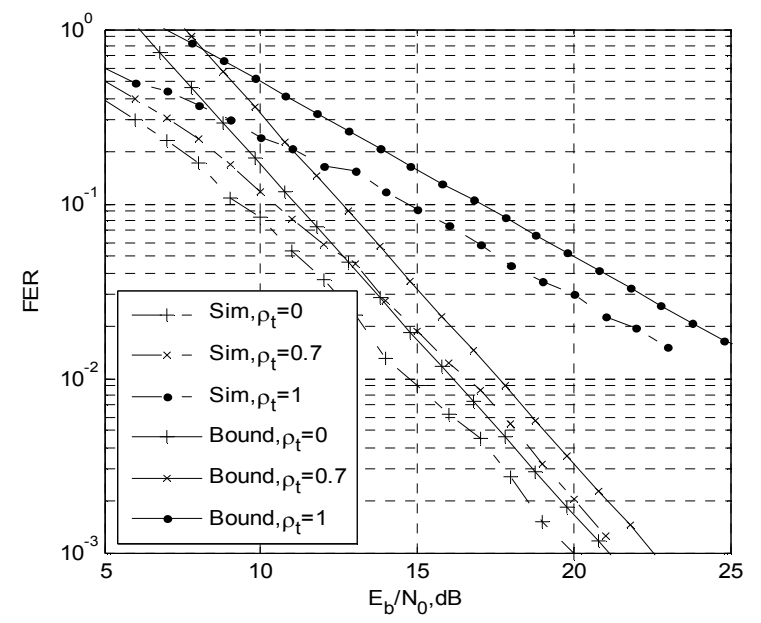

Fig. 2: FER performance of OST-4CPM with two transmit antennas and one receive antenna

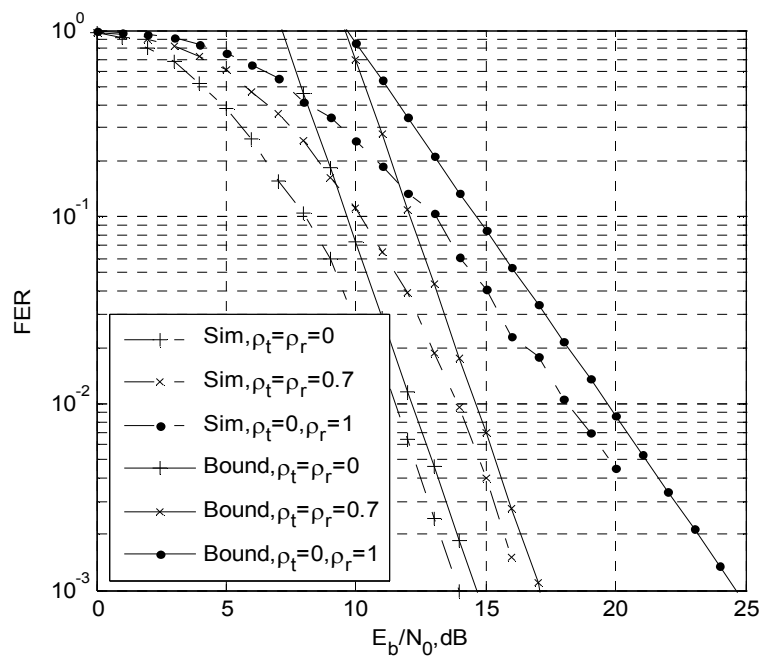

Fig. 2: FER performance of OST-4CPM with two transmit antennas and two receive antennas

results of this algorithm are plotted against $\mathrm{E}_{\mathrm{b}} / \mathrm{N}_{0}$. OSTCPM systems of full response CPM signals with two transmit antennas are considered. The number of receive antennas is one and two. The modulation level $\mathrm{M}$ is chosen to be 4, while the modulation index $\mathrm{h}=$ $1 / \mathrm{M}$. The pulse shaping functions $\mathrm{g}(\mathrm{t})$ and $\mathrm{g}_{0}(\mathrm{t})$ are chosen to be 1REC and 1RC, respectively. The transmit and receive antenna correlation matrices are given by:

$$
R_{T}=\left[\begin{array}{cc}
1 & \rho_{t} \\
\rho_{t} & 1
\end{array}\right], R_{R}=\left[\begin{array}{cc}
1 & \rho_{r} \\
\rho_{r} & 1
\end{array}\right]
$$

for the two transmit and two receive antennas system.

Figure 2 and 3 show the FER performance of the MLSD algorithm for the OST-4CPM system over Rayleigh fading channels. It can be seen from the figures that the system in uncorrelated MIMO channels $\left(\rho_{t}=\rho_{r}=0\right)$ has the same diversity gain as that of the system over partially correlated channels $\left(0<\rho_{t}<1\right.$, $\left.0<\rho_{r}<1\right)$. When the channel is fully correlated the diversity gain decreases, which matches well with the theoretical analysis. From Fig. 2 we can see that the performance over a correlated channel is degraded by approximate $2 \mathrm{~dB}$ at a FER of $10^{-2}$, when the correlated indexes $\rho_{t}$ increases from 0 to 0.7 . And the penalty on the code performance increases to over $10 \mathrm{~dB}$ at the same FER in fully correlated channel. It can also be observed from the two figures that the upper bounds are looser when the diversity is low, e.g., if the number of receive antennas is small, or the transmit or receive antennas are fully correlated.

\section{CONCLUSION}

The effect of the spatial correlation on MLSD of OST-CPM system over Rayleigh fading channels was investigated in the study. The PWEP upper bound of OST-CPM systems was deduced to evaluate the achievable diversity gain over quasi-static Rayleigh fading channels with spatial correlation. Due to the orthogonality and the constant envelope, the OST-CPM system in partially correlated channels can achieve full diversity. The simulation results well match the theoretical analysis.

\section{REFERENCES}

Altamonte, S., 1998. A simple transmit diversity technique for wireless communications. IEEE J. Sel. Area Comm., 16(8): 1451-1458.

Anderson, J., C. Sandburg and T. Aalen, 1986. Digital Phase Modulation. Springer, ISBN: 030642195X; ISBN-13: 9780306421952.

Aulin, T., N. Rydbeck and C. Sandburg, 1981. Continuous phase modulation- Part I and Part II. IEEE T. Commun., 29(8): 196-225.

Baro, S., G. Bauch and A. Hansmann, 2000. Improved codes for space-time trellis-coded modulation. IEEE Commun. Lett., 4(1): 20-22.

Bolcskei, H. and A.P. Raj, 2000. Performance of spacetime codes in the presence of spatial fading correlation. Proceeding of Conference Record of the 34th Asilomar Conference on Signals, Systems and Computers, Pacific Grove, CA, USA.

Chuah, C., D. Tse, J. Kahn and R. Valenzuela, 2002. Capacity scaling in MIMO wireless systems under correlated fading. IEEE T. Inform. Theory, 48(3): 637-650.

Foschini, G., 1996. Layered space-time architecture for wireless communication in a fading environment when using multiple antennas. Bell Lab Tech. J., 1(2): 41-59. 
Hedayat, A., H. Shah and A. Nosratinia, 2005. Analysis of space-time coding in correlated fading channels. IEEE T. Wirel. Commun., 4(6): 2882-2891.

Hesse, M., J. Lebrun and L. Deneire, 2011. L2orthogonal ST-code design for CPM. IEEE $\mathrm{T}$. Commun., 59(11): 3158-3166.

Hochwald, B.M. and T.L. Marzetta, 2000. Unitary space-time modulation for multipleantenna communications in Rayleigh flat fading. IEEE $\mathrm{T}$. Inform. Theory, 46(2): 543-564.

Hughes, B.L., 2000. Differential space-time modulation. IEEE T. Inform. Theory, 46(7): 2567-2578.

Naguib, A., V. Tarokh, N. Seshadri and A. Calderbank, 1998. A space-time coding modem for high-datarate wireless communications. IEEE J. Sel. Area Comm., 16(8): 1459-1478.

Proakis, J., 1983. Digital Communications. 2nd and 3rd Edn., McGraw-Hill, New York.

Tarokh, V., N. Seshadri and A. Calderbank, 1998. Space-time codes for high data rate wireless communication: Performance criterion and code construction. IEEE T. Inform. Theory, 44(2): 744-765.

Tarokh, V., A. Naguib, N. Seshadri and A. Calderbank, 1999a. Space-time codes for high data rate wireless communication: Performance criteria in the presence of channel estimation errors, mobility and multiple paths. IEEE T. Commun., 47(2): 199-207.
Tarokh, V., H. Jafarkhani and A.R. Calderbank, 1999b. Space-time block codes from orthogonal designs. IEEE T. Inform. Theory, 45(5): 1456-1467.

Uysal, M. and C. Georghiades, 2001. Effect of spatial fading correlation on performance of space-time codes. Elect. Lett., 37(3): 181-183.

Wang, G. and X. Xia, 2004. An orthogonal space-time coded CPM system with fast decoding for two transmit antennas. IEEE T. Inform. Theory, 3: 486-493.

Wang, W.W. and S.S. Abeysekera, 2011. Novel block construction using tail sequences for space time block coded CPM information. Proceeding of 8th International Conference on Communications and Signal Processing (ICICS), Singapore, pp: 1-5.

Wang, D., G. Wang and X. Xia, 2005. An orthogonal space-time coded partial response CPM system with fast decoding for two transmit antennas. IEEE T. Wirel. Commun., 4(5): 2410-2422.

Zajic, A.G. and G.L. Stuber, 2009. A space-time code design for CPM: Diversity order and coding gain. IEEE T. Inform. Theory, 55(8): 3781-3798.

Zhang, X.X. and M. Fitz, 2003. Space-time code design with continuous phase modulation. IEEE J. Sel. Area Comm., 21(5): 783-792. 